\title{
Non-thermal production of pNGB dark matter and inflation
}

\author{
Yoshihiko Abe, ${ }^{a}$ Takashi Toma $^{b}$ and Koichi Yoshioka ${ }^{a}$ \\ ${ }^{a}$ Department of Physics, Kyoto University, \\ Kyoto 606-8502, Japan \\ ${ }^{b}$ Institute of Liberal Arts and Science, \\ Kanazawa University, Kakuma-machi, Kanazawa 920-1192, Japan \\ E-mail: y.abe@gauge.scphys.kyoto-u.ac.jp, \\ toma@staff.kanazawa-u.ac.jp, yoshioka@gauge.scphys.kyoto-u.ac.jp
}

ABSTRACT: A pseudo Nambu-Goldstone boson (pNGB) is a natural candidate of dark matter in that it avoids the severe direct detection bounds. We show in this paper that the pNGB has another different and interesting face with a higher symmetry breaking scale. Such large symmetry breaking is motivated by various physics beyond the standard model. In this case, the pNGB interaction is suppressed due to the Nambu-Goldstone property and the freeze-out production does not work even with sufficiently large portal coupling. We then study the pNGB dark matter relic abundance from the out-of-equilibrium production via feeble Higgs portal coupling. Further, a possibility is pursued the symmetry breaking scalar in the pNGB model plays the role of inflaton. The inflaton and dark matter are unified in a single field and the pNGB production from inflaton decay is inevitable. For these non-thermally produced relic abundance of pNGB dark matter and successful inflation, we find that the dark matter mass should be less than a few $\mathrm{GeV}$ in the wide range of the reheating temperature and the inflaton mass.

Keywords: Beyond Standard Model, Cosmology of Theories beyond the SM

ArXiv ePrint: 2012.10286 


\section{Contents}

1 Introduction 1

2 pNGB dark matter model 2

3 pNGB production via freeze-in $\quad 4$

3.1 Boltzmann equations 4

$\begin{array}{ll}3.2 & \text { Thermal mass of the Higgs boson }\end{array}$

$\begin{array}{lll}3.3 & \text { IR freeze-in }\left(T_{R} \gg m_{\phi}\right) & 7\end{array}$

3.4 UV freeze-in $\left(T_{R} \ll m_{\phi}\right) \quad 9$

4 Inflation $\quad 10$

$\begin{array}{ll}\text { 4.1 Inflationary dynamics and constraints } & 10\end{array}$

$\begin{array}{lll}4.2 & \text { pNGB production from inflaton } & 12\end{array}$

$\begin{array}{lll}4.3 & \text { Dark matter abundance } & 14\end{array}$

$\begin{array}{llr}5 & \text { Conclusion } & 17\end{array}$

$\begin{array}{ll}\text { A Thermal mass contribution } & 18\end{array}$

\section{Introduction}

Revealing the evolution of the universe is a key subject not only for cosmology but also for particle physics. In particular, in the situation that there is no clear signature of physics beyond the Standard Model (SM) at the Large Hadron Collider so far, exploring nature of dark matter which is presumed to exist in the universe from various observations can give substantial hints for physics beyond the SM.

One of the well-motivated dark matter candidates is so-called WIMPs (Weakly Interacting Massive Particles) which are thermally produced in the early universe via sufficient interactions with the SM particles. WIMPs are widely being searched by various experiments through indirect detection, direct detection, particle collider experiments and astrophysical observations. However there is no clear evidence for WIMPs so far, and the resultant experimental constraints become stronger and stronger. Direct detection experiments especially give strong constraints on interactions between dark matter and nuclei. The current upper bound in terms of WIMP-nucleon spin-independent cross section is $4.1 \times 10^{-47} \mathrm{~cm}^{2}$ at $30 \mathrm{GeV}$ WIMP mass, which is given by the XENON1T Collaboration [1]. In addition, as future sensitivity, the XENONnT experiment is expected to update the bound to $2.6 \times 10^{-48} \mathrm{~cm}^{2}$ at $50 \mathrm{GeV}$ WIMP mass [2]. Such a severe constraint may imply that the interactions between dark matter and SM particles are rather weak, which means less motivation for thermal WIMP paradigm. 
One of the ways naturally evading the severe constraints from direct detection is to identify a pseudo Nambu-Goldstone boson (pNGB) as dark matter. The simplest model of pNGB dark matter with global $\mathrm{U}(1)$ symmetry breaking has been proposed in ref. [3] and its extensions with gauged $\mathrm{U}(1)_{B-L}$ symmetry are discussed in refs. [4, 5]. In addition, various aspects of the pNGB dark matter model, such as a global fit [6], have been studied. In these cases, it is found due to the nature of NGB that all couplings of dark matter are inversely proportional to the vacuum expectation value (VEV) associated with the symmetry breaking, and then highly suppressed if the VEV is large enough.

Such a large VEV may in fact be connected to generate the small neutrino masses in the framework of Majoron models where the right-handed neutrino Majorana masses are induced by the large VEV [7-12]. In this case, the pNGB is identified as Majoron. In order to make the canonical seesaw mechanism work with $\mathcal{O}(1)$ Yukawa couplings, the VEV should be as large as $\mathcal{O}\left(10^{14}\right) \mathrm{GeV}$. Therefore from this viewpoint, it is motivated to consider the pNGB as FIMPs (Feebly Interacting Massive Particles) produced by freeze-in mechanism [13-15] with extremely suppressed interactions, e.g., due to a large VEV. In the framework of freeze-in mechanism, dark matter is assumed to be never thermalized with the SM particles. A typical magnitude of FIMP coupling for reproducing the relic abundance observed by the PLANCK Collaboration [16] is $\mathcal{O}\left(10^{-11}\right)$ for dimensionless couplings [15].

In this paper, we calculate the dark matter relic abundance via Higgs portal in the pNGB dark matter model [3] with large symmetry breaking scale, and study in detail the freeze-in parameter space consistent with the observations. In addition, we examine if successful inflation can occur through the non-minimal coupling to gravity where the field associated with the symmetry breaking is identified as the inflaton. That implies the inflaton also necessarily induces the pNGB dark matter relic, which would significantly modify the relevant parameter space.

The rest part of this paper is organized as follows. In section 2, we briefly review the pNGB model. In section 3, the relic abundance of pNGB dark matter via the Higgs portal freeze-in is calculated, including the thermal mass of the Higgs boson which is important to evaluate the reaction rates of relevant processes. We also derive the Boltzmann equations for the pNGB FIMP, evaluate the time evolution of the dark matter yield, and show some parameter sets consistent with the present relic abundance observed by experiments. In section 4, we examine the possibility that the radial component of symmetry breaking scalar plays an role of the inflaton. The allowed parameter space is identified taking into account the direct production of the pNGB dark matter from the inflaton decay. Section 5 is devoted to our conclusion.

\section{2 pNGB dark matter model}

In the pNGB model, the SM is extended with a complex singlet scalar $\Phi$, and the Lagrangian is given by

$$
\mathcal{L}=\mathcal{L}_{\mathrm{SM}}+\left|\partial_{\mu} \Phi\right|^{2}-\mathcal{V}(H, \Phi)
$$


where the scalar potential including the SM Higgs doublet $H$ is given by

$$
\begin{aligned}
\mathcal{V}(H, \Phi)= & -\frac{\mu_{H}^{2}}{2}|H|^{2}+\frac{\lambda_{H}}{2}|H|^{4}-\frac{\mu_{\Phi}^{2}}{2}|\Phi|^{2}+\frac{\lambda_{\Phi}}{2}|\Phi|^{4}+\lambda_{H \Phi}|H|^{2}|\Phi|^{2} \\
& -\frac{m^{2}}{4}\left(\Phi^{2}+\Phi^{* 2}\right) .
\end{aligned}
$$

The last term is the soft-breaking mass term which is introduced in order to generate the mass of pNGB. We do not consider the origin of this term (ultraviolet (UV) completion of the model), while some examples have been discussed in the literature $[4,5]$. The Higgs doublet $H$ and the singlet scalar $\Phi$ are assumed to develop non-vanishing VEVs and are parametrized as

$$
H=\frac{1}{\sqrt{2}}\left(\begin{array}{c}
0 \\
v+h
\end{array}\right), \quad \Phi=\frac{v_{\phi}+\phi}{\sqrt{2}} e^{i \chi / v_{\phi}}
$$

where we have dropped the would-be NG modes in $H$ (the unitary gauge). Note that the pNGB $\chi$ is stable due to a remnant $\mathbb{Z}_{2}$ symmetry after the spontaneous symmetry breaking, thus it can be a dark matter candidate. The stationary conditions of the VEVs $v$ and $v_{\phi}$ impose the following relations between the parameters in the scalar potential

$$
\mu_{H}^{2}=\lambda_{H} v^{2}+\lambda_{H \Phi} v_{\phi}^{2}, \quad \mu_{\Phi}^{2}=\lambda_{\Phi} v_{\phi}^{2}+\lambda_{H \Phi} v^{2}-m^{2} .
$$

Using these relations, the masses of the scalar fields are evaluated in the following two phases:

- The electroweak symmetry is unbroken, $\langle H\rangle=0$ and $\langle\Phi\rangle=v_{\phi} / \sqrt{2}$. In this phase, only the components of $\Phi$ acquire the masses as

$$
m_{\phi}^{2}=\lambda_{\Phi} v_{\phi}^{2}, \quad m_{\chi}^{2}=m^{2} .
$$

- The electroweak symmetry is spontaneously broken, $\langle H\rangle=(0, v / \sqrt{2})^{\mathrm{T}}$ and $\langle\Phi\rangle=$ $v_{\phi} / \sqrt{2}$. In this phase, the physical component of $H$ is massive and the mass eigenvalues of the scalar fields are given by

$$
\begin{aligned}
m_{h_{1}}^{2} & =\frac{1}{2}\left[\lambda_{H} v^{2}+\lambda_{\Phi} v_{\phi}^{2}-\sqrt{\left(\lambda_{\Phi} v_{\phi}^{2}-\lambda_{H} v^{2}\right)^{2}+4 \lambda_{H \Phi}^{2} v^{2} v_{\phi}^{2}}\right], \\
m_{h_{2}}^{2} & =\frac{1}{2}\left[\lambda_{H} v^{2}+\lambda_{\Phi} v_{\phi}^{2}+\sqrt{\left(\lambda_{\Phi} v_{\phi}^{2}-\lambda_{H} v^{2}\right)^{2}+4 \lambda_{H \Phi}^{2} v^{2} v_{\phi}^{2}}\right], \\
m_{\chi}^{2} & =m^{2} .
\end{aligned}
$$

The lighter CP-even scalar $h_{1}$ is identified as the SM-like Higgs boson with the mass $125 \mathrm{GeV}$. The mixing angle of the CP-even scalars is given by

$$
\tan 2 \alpha=\frac{2 \lambda_{H \Phi} v v_{\phi}}{\lambda_{\Phi} v_{\phi}^{2}-\lambda_{H} v^{2}}
$$


which is introduced as

$$
\left(\begin{array}{l}
h \\
\phi
\end{array}\right)=\left(\begin{array}{cc}
\cos \alpha & \sin \alpha \\
-\sin \alpha & \cos \alpha
\end{array}\right)\left(\begin{array}{l}
h_{1} \\
h_{2}
\end{array}\right) .
$$

Note that the mixing can safely be ignored in our setup with a large hierarchy between the VEVs $\left(v \ll v_{\phi}\right)$.

In this paper, we mainly use the non-linear representation for the fluctuations of $\Phi$ field as given in eq. (2.3). The same physics is obtained also in the linear representation. The Lagrangian in the broken phase of $\Phi$ contains

$$
\begin{aligned}
\mathcal{L} \supset & \frac{1}{2}\left[\left(\partial_{\mu} \phi\right)^{2}-m_{\phi}^{2} \phi^{2}\right]+\frac{1}{2}\left(1+\frac{\phi}{v_{\phi}}\right)^{2}\left[\left(\partial_{\mu} \chi\right)^{2}-m_{\chi}^{2} v_{\phi}^{2} \sin ^{2}\left(\frac{\chi}{v_{\phi}}\right)\right] \\
& -\frac{\lambda_{\Phi}}{8} \phi^{4}-\frac{\lambda_{\Phi} v_{\phi}}{2} \phi^{3}-\frac{\lambda_{H \Phi}}{2}|H|^{2}\left(v_{\phi}+\phi\right)^{2} .
\end{aligned}
$$

The interaction terms of pNGB dark matter $\chi$ are originated from the scalar kinetic term and the $\mathrm{U}(1)$ soft-breaking mass term in this representation.

\section{3 pNGB production via freeze-in}

In this section, we discuss how the pNGB dark matter relics are produced via the freeze-in mechanism and the parameter set consistent with the observations. We assume that the relic abundance of dark matter is determined in the radiation dominant era of the universe, in which the Hubble parameter $H$ and entropy density $s$ are given as the functions of the temperature $T$ as

$$
H=\sqrt{\frac{\pi^{2}}{90} g_{*}} \frac{T^{2}}{M_{P}}, \quad s=\frac{2 \pi^{2}}{45} g_{*}^{S} T^{3},
$$

where $g_{*}$ and $g_{*}^{S}$ denote the total numbers of effective massless degrees of freedom contributing to the energy and entropy densities, respectively [17], and $M_{P}$ is the reduced Planck mass $M_{P}=1 / \sqrt{8 \pi G_{N}}=2.4 \times 10^{18} \mathrm{GeV}\left(G_{N}\right.$ is the gravitational constant).

\subsection{Boltzmann equations}

We are interested in the case that both of dark matter $\chi$ and the CP-even scalar $\phi\left(\simeq h_{2}\right)$ are never thermalized with the SM particles. This is achieved by tiny values of quartic couplings $\lambda_{H \Phi}$ and $\lambda_{\Phi}$, roughly speaking, $\lambda_{H \Phi}, \lambda_{\Phi} \lesssim 10^{-6}$. From the theoretical side, a radiative correction to the $|\Phi|^{4}$ term would imply its lower bound, $\lambda_{\Phi} \gtrsim \lambda_{H \Phi}^{2} / 16 \pi^{2}$. With these feeble couplings, both of $\phi$ and $\chi$ become the FIMPs, and the Boltzmann equations for the number densities $n_{\phi}$ and $n_{\chi}$ are given by

$$
\begin{aligned}
& \frac{d n_{\phi}}{d t}+3 H n_{\phi}=C_{H^{\dagger} H \leftrightarrow \phi \phi}+C_{\chi \chi \leftrightarrow \phi \phi}+C_{H^{\dagger} H \leftrightarrow \phi}+C_{\chi \chi \leftrightarrow \phi}, \\
& \frac{d n_{\chi}}{d t}+3 H n_{\chi}=C_{\phi \phi \leftrightarrow \chi \chi}+C_{H^{\dagger} H \leftrightarrow \chi \chi}+C_{\phi \leftrightarrow \chi \chi},
\end{aligned}
$$


where $C_{A \leftrightarrow B}$ in the right-hand side denotes the collision term corresponding to the process $A \leftrightarrow B$. Here the broken phase of $\Phi$ is assumed and the scalars in the dark sector interact with the SM only through the Higgs doublet. The explicit form of collision terms is

$$
\begin{aligned}
C_{i j \ldots \leftrightarrow a b \ldots}= & \int \prod_{i} d \Pi_{i} f_{i} \prod_{a} d \Pi_{a}\left(1+f_{a}\right)(2 \pi)^{4} \delta^{4}\left(\sum_{i} p_{i}-\sum_{a} p_{a}\right)\left|\mathcal{M}_{i j \ldots \rightarrow a b \ldots}\right|^{2} \\
& -\int \prod_{a} d \Pi_{a} f_{a} \prod_{i} d \Pi_{i}\left(1+f_{i}\right)(2 \pi)^{4} \delta^{4}\left(\sum_{a} p_{a}-\sum_{i} p_{i}\right)\left|\mathcal{M}_{a b \ldots \rightarrow i j \ldots}\right|^{2},
\end{aligned}
$$

where $f_{x}$ is the distribution function of particle $x, d \Pi_{x}$ is the Lorentz-invariant phase space expressed as $d \Pi_{x}=\frac{d^{3} p_{x}}{(2 \pi)^{3} 2 E_{p_{x}}}$, and $\mathcal{M}_{X}$ denotes the amplitude of the process $X$.

Since dark matter is produced by the freeze-in mechanism from the SM thermal bath, the magnitude of distribution functions is tiny for $\phi$ and $\chi$. Thus the $\mathcal{O}\left(f_{\phi, \chi}^{2}\right)$ terms can be dropped in the above equations. This approximation is valid as long as the distribution functions $f_{\phi, \chi}$ are not close to the equilibrium one before the abundance is frozen. The Boltzmann equations are thus reduced to

$$
\begin{aligned}
& \frac{d n_{\phi}}{d t}+3 H n_{\phi}=C_{H^{\dagger} H \rightarrow \phi \phi}+C_{H^{\dagger} H \rightarrow \phi}-\int d \Pi_{\phi} f_{\phi}\left(\boldsymbol{p}_{\phi}\right) 2 m_{\phi}\left(\Gamma_{\phi \rightarrow H^{\dagger} H}+\Gamma_{\phi \rightarrow \chi \chi}\right), \\
& \frac{d n_{\chi}}{d t}+3 H n_{\chi}=C_{H^{\dagger} H \rightarrow \chi \chi}+2 \int d \Pi_{\phi} f_{\phi}\left(\boldsymbol{p}_{\phi}\right) 2 m_{\phi} \Gamma_{\phi \rightarrow \chi \chi},
\end{aligned}
$$

where the decay widths are given by

$$
\Gamma_{\phi \rightarrow H^{\dagger} H}=\frac{\lambda_{H \Phi}^{2} m_{\phi}}{8 \pi \lambda_{\Phi}} \sqrt{1-\frac{4 m_{H}^{2}}{m_{\phi}^{2}}}, \quad \Gamma_{\phi \rightarrow \chi \chi}=\frac{\lambda_{\Phi} m_{\phi}}{32 \pi} \sqrt{1-\frac{4 m_{\chi}^{2}}{m_{\phi}^{2}}} .
$$

Note that we have used the relation $m_{\phi}^{2}=\lambda_{\Phi} v_{\phi}^{2}$ in the above equations and the SM Higgs doublet contains real four components, leading to the difference of numerical factors. The mass parameter $m_{H}$ for the $H$ field will be discussed in the next section. Introducing the net dark matter number density $n_{D}=n_{\chi}+2 \mathrm{Br}^{\phi \rightarrow \chi \chi} n_{\phi}$, the Boltzmann equation for $n_{D}$ is recast as

$$
\frac{d n_{D}}{d t}+3 H n_{D}=C_{H^{\dagger} H \rightarrow \chi \chi}+2 \mathrm{Br}^{\phi \rightarrow \chi \chi}\left(C_{H^{\dagger} H \rightarrow \phi \phi}+C_{H^{\dagger} H \rightarrow \phi}\right)
$$

where $\mathrm{Br}^{\phi \rightarrow \chi \chi}$ is the branching ratio defined by $\mathrm{Br}^{\phi \rightarrow \chi \chi}=\Gamma_{\phi \rightarrow \chi \chi} /\left(\Gamma_{\phi \rightarrow H^{\dagger} H}+\Gamma_{\phi \rightarrow \chi \chi}\right)$. The collision terms are written by using the thermally averaged cross sections and the number density of the SM Higgs doublet in thermal bath, $n_{H}^{\mathrm{eq}}$. Then we obtain

$$
\frac{d n_{D}}{d t}+3 H n_{D}=2\left[\left\langle\sigma_{H^{\dagger} H \rightarrow \chi \chi} \bar{v}\right\rangle+2 \operatorname{Br}^{\phi \rightarrow \chi \chi}\left\langle\sigma_{H^{\dagger} H \rightarrow \phi \phi} \bar{v}\right\rangle+\operatorname{Br}^{\phi \rightarrow \chi \chi}\left\langle\sigma_{H^{\dagger} H \rightarrow \phi} \bar{v}\right\rangle\right]\left(n_{H}^{\mathrm{eq}}\right)^{2} .
$$

The first term in the right-hand side denotes the dark matter production directly from the thermal bath, and the second and third terms are the contributions from the decays of 
$\phi$ produced from the thermal bath. The thermally averaged cross sections are explicitly calculated as

$$
\begin{aligned}
\left\langle\sigma_{H^{\dagger} H \rightarrow \chi \chi} \bar{v}\right\rangle\left(n_{H}^{\mathrm{eq}}\right)^{2} & =\frac{\lambda_{H \Phi}^{2} T^{4}}{256 \pi^{5}} \int_{2 \bar{x}_{\chi}}^{\infty} d z \sqrt{z^{2}-4 x_{\chi}^{2}} \sqrt{z^{2}-4 x_{H}^{2}} \frac{z^{4} K_{1}(z)}{\left(z^{2}-x_{\phi}^{2}\right)^{2}+x_{\phi}^{2} \gamma_{\phi}^{2}}, \\
\left\langle\sigma_{H^{\dagger} H \rightarrow \phi \phi} \bar{v}\right\rangle\left(n_{H}^{\mathrm{eq}}\right)^{2} & =\frac{\lambda_{H \Phi}^{2} T^{4}}{256 \pi^{5}} \int_{2 \bar{x}_{\phi}}^{\infty} d z \sqrt{z^{2}-4 x_{\phi}^{2}} \sqrt{z^{2}-4 x_{H}^{2}} \frac{\left(z^{2}+2 x_{\phi}^{2}\right)^{2}+x_{\phi}^{2} \gamma_{\phi}^{2}}{\left(z^{2}-x_{\phi}^{2}\right)^{2}+x_{\phi}^{2} \gamma_{\phi}^{2}} K_{1}(z), \\
\left\langle\sigma_{H^{\dagger} H \rightarrow \phi} \bar{v}\right\rangle\left(n_{H}^{\mathrm{eq}}\right)^{2} & =\frac{\lambda_{H \Phi}^{2} m_{\phi}^{3} T}{16 \pi^{3} \lambda_{\Phi}} K_{1}\left(x_{\phi}\right) \sqrt{1-\frac{4 m_{H}^{2}}{m_{\phi}^{2}}}
\end{aligned}
$$

where we have defined the integral variable $z \equiv \sqrt{s} / T$ for the Mandelstam $s$ variable, the dimensionless parameters $x_{i} \equiv m_{i} / T$ and $\gamma_{i} \equiv \Gamma_{i} / T$ for a particle $i$ with the mass $m_{i}$ and the total decay width $\Gamma_{i}$. In the integrals, the lower limits are given by $\bar{x}_{\chi, \phi}=$ $\max \left[x_{\chi, \phi}, x_{H}\right]$ and $K_{1}(z)$ is the modified Bessel function of second kind of order 1. Here thermal bath particles are assumed to obey the Maxwell-Boltzmann distribution. ${ }^{1}$ Note that the integrand in eq. (3.10) contains the factor $z^{4}$ due to the derivative coupling of pNGB dark matter. This behavior implies that even if the portal coupling $\lambda_{H \Phi}$ is large, the dark matter reaction rate with the SM is suppressed in lower energy than $m_{\phi}$ and the usual freeze-out does not work in the case of the hierarchical VEV $v_{\phi} \gg v\left(m_{\phi} \gg m_{h}\right)$.

\subsection{Thermal mass of the Higgs boson}

The one-loop effect of bath particles leads to the mass corrections quadratically scaling by the temperature, which are called thermal masses. Including thermal mass corrections gives important effects for collision terms (reaction rates) and hence for the evaluation of relic abundance of dark matter. The detailed calculation of thermal mass for the SM Higgs boson is summarized in appendix A. The electroweak gauge bosons, all SM fermions, and all components of $H$ including the NGBs eaten by the gauge bosons contribute to the thermal mass, which is given by

$$
\Delta_{h}=\left(\frac{g_{Y}^{2}}{16}+\frac{3 g_{2}^{2}}{16}+\frac{y_{t}^{2}}{4}+\frac{\lambda_{H}}{4}\right) T^{2}
$$

where $g_{Y}$ and $g_{2}$ are the gauge couplings of $\mathrm{U}(1)_{Y}$ and $\mathrm{SU}(2)_{L}$, and $y_{t}$ is the top Yukawa coupling which is dominant over the other Yukawa couplings. Including this thermal contribution, the mass of the SM Higgs boson is given by

$$
m_{H}^{2}=m_{h_{1}}^{2}+\Delta_{h}
$$

which plays a role of regulator in the reaction rates in the Boltzmann equations.

\footnotetext{
${ }^{1}$ Quantum statistical distributions may give a small factor difference in numerical calculations [18].
} 

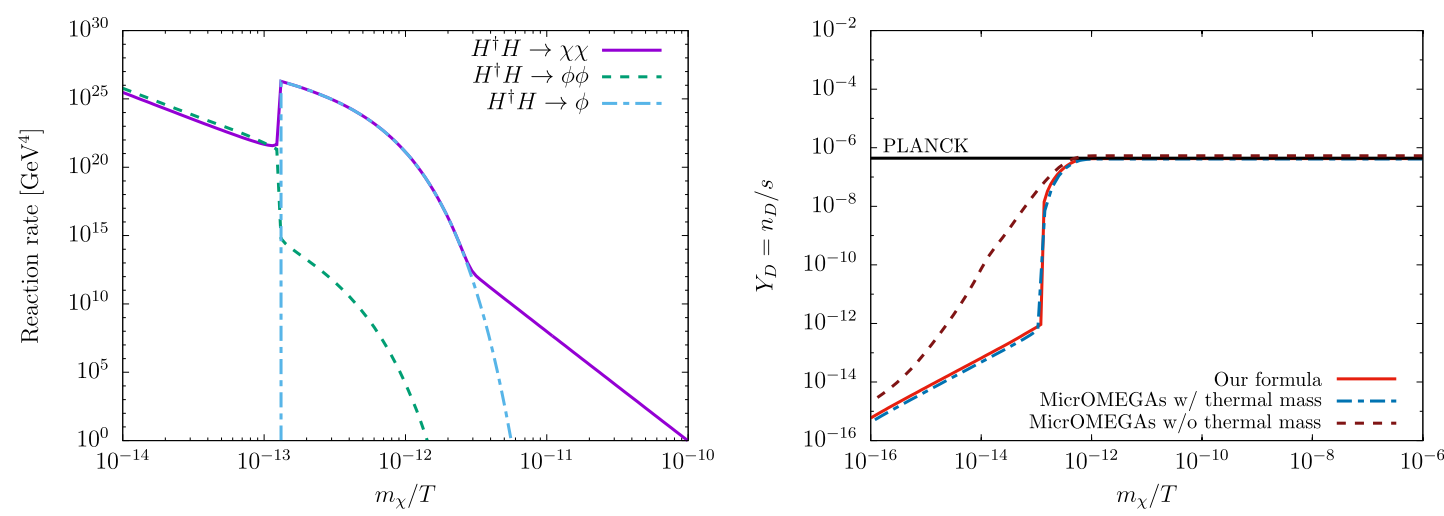

Figure 1. Left: evolution of the reaction rates for three processes eqs. (3.10)-(3.12). Right: evolution of the dark matter yield determined by solving the Boltzmann equation. The red solid, dot-dashed dark blue and dashed brown lines denote the results using our formula (3.9), the public code MicrOMEGAs with and without the thermal mass effect for the SM Higgs boson, respectively. The black horizontal line corresponds to the correct dark matter abundance for $m_{\chi}=1 \mathrm{MeV}$.

\subsection{IR freeze-in $\left(T_{R} \gg m_{\phi}\right)$}

When the reheating temperature of the universe, $T_{R}$, is higher than the heavy mediator mass $m_{\phi}$, the final abundance of dark matter is determined independently of the reheating temperature. This is so-called the IR freeze-in [15]. The analytic formula of the dark matter yield $Y_{D}=n_{D} / s$ derived from eq. (3.9) is approximately given by

$$
Y_{D}^{\mathrm{IR}} \approx \frac{405 \sqrt{10}}{(2 \pi)^{5}} \frac{\mathrm{Br}^{\phi \rightarrow \chi \chi} \lambda_{H \Phi}^{2} M_{P}}{g_{*}^{S} g_{*}^{1 / 2} \lambda_{\Phi} m_{\phi}} \sqrt{1-\frac{4 m_{h}^{2}}{m_{\phi}^{2}}} .
$$

The result is found to be independent of the dark matter mass because the dominant production process comes from the $\phi$ decay around $T \sim m_{\phi}$, as shown in the following part. In the limit where the scalar VEV and the mass of $\phi$ are much larger than the masses of the SM Higgs boson and dark matter, the factor of quartic couplings $\mathrm{Br}^{\phi \rightarrow \chi \chi} \lambda_{H \Phi}^{2}$ reduces to

$$
\mathrm{Br}^{\phi \rightarrow \chi \chi} \lambda_{H \Phi}^{2} \approx \frac{\lambda_{\Phi}^{2} \lambda_{H \Phi}^{2}}{\lambda_{\Phi}^{2}+4 \lambda_{H \Phi}^{2}} \approx\left\{\begin{array}{ll}
\lambda_{H \Phi}^{2} & \text { for } \lambda_{\Phi} \gg \lambda_{H \Phi} \\
\frac{\lambda_{\Phi}^{2}}{4} & \text { for } \lambda_{\Phi} \ll \lambda_{H \Phi}
\end{array} .\right.
$$

Thus combining the IR freeze-in relic eq. (3.15) and the yield corresponding to the observed value $Y_{D}^{\text {obs. }}=4.4 \times 10^{-7}\left(m_{\chi} / \mathrm{MeV}\right)^{-1}[16]$ with $g_{*} \sim g_{*}^{S} \sim 100$, we obtain the following relations

$$
\begin{aligned}
\left(\frac{\lambda_{\Phi}}{10^{-8}}\right) & \approx\left(\frac{\lambda_{H \Phi}}{2 \times 10^{-9}}\right)^{2}\left(\frac{m_{\chi}}{1 \mathrm{MeV}}\right)\left(\frac{10^{10} \mathrm{GeV}}{m_{\phi}}\right) & & \text { for } \lambda_{\Phi} \gg \lambda_{H \Phi}, \\
\left(\frac{\lambda_{\Phi}}{7 \times 10^{-11}}\right) & \approx\left(\frac{1 \mathrm{MeV}}{m_{\chi}}\right)\left(\frac{m_{\phi}}{10^{10} \mathrm{GeV}}\right) & & \text { for } \lambda_{\Phi} \ll \lambda_{H \Phi} .
\end{aligned}
$$



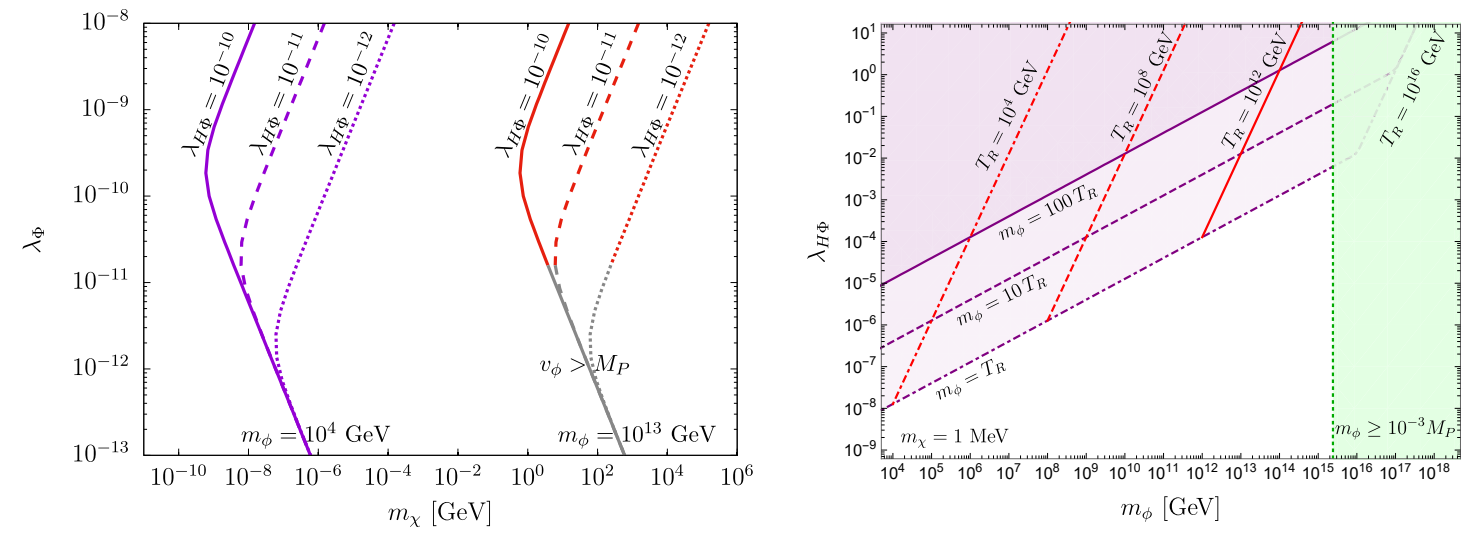

Figure 2. Left: contours reproducing the observed relic abundance in the $\left(m_{\chi}, \lambda_{\Phi}\right)$ plane where the mediator mass is fixed to be $m_{\phi}=10^{4} \mathrm{GeV}\left(10^{13} \mathrm{GeV}\right)$ in the purple (red) line. The solid, dashed and dotted lines correspond to $\lambda_{H \Phi}=10^{-10}, 10^{-11}$ and $10^{-12}$, respectively. The gray parts of the red lines are excluded by the criterion $v_{\phi}>M_{P}$. Right: the purple region can realize the observed relic abundance via the UV freeze-in in the $\left(m_{\phi}, \lambda_{H \Phi}\right)$ plane. Each contour reproduces the abundance for a specific parameter set. The solid, dashed and dot-dashed red lines correspond to $T_{R}=10^{12} \mathrm{GeV}, 10^{8} \mathrm{GeV}$ and $10^{4} \mathrm{GeV}$, respectively. The green region, $m_{\phi} \geq 10^{-3} M_{P}$, is excluded (see the text).

The evolution of the reaction rates including the thermal mass effects is shown in the left panel of figure 1 where we choose the following parameter set

$$
\lambda_{\phi}=7 \times 10^{-11}, \quad \lambda_{H \Phi}=10^{-7}, \quad m_{\chi}=1 \mathrm{MeV}, \quad m_{\phi}=10^{10} \mathrm{GeV},
$$

as a benchmark. This is chosen so that the parameter relation eq. (3.18) is realized. Note that the portal coupling should satisfy $\lambda_{H \Phi} \lesssim 10^{-6}$ in order for the dark sector particles not to enter into the thermal bath. When the temperature cools down to the mediator mass scale $T \sim m_{\phi}$, a number of on-shell $\phi$ are resonantly produced. As a result the magnitude of the reaction rate for the process $H^{\dagger} H \rightarrow \chi \chi$ rapidly increases as can be seen in figure 1 .

For the same benchmark parameter set, the evolution of the dark matter yield is shown in the right panel of figure 1, assuming the vanishing initial conditions of the dark sector

$$
Y_{\chi}\left(T=T_{R}\right)=Y_{\phi}\left(T=T_{R}\right)=0 .
$$

We also show for comparison the results calculated by the public code MicrOMEGAs [19] with and without the thermal mass effect for the SM Higgs boson. ${ }^{2}$ As obvious from the plot, the thermal mass effect gives an impact on the evolution of the dark matter yield, in particular when the temperature is $T \gtrsim m_{\phi}$. Including thermal mass also affects the final dark matter abundance, while its impact is not so large and only gives a few factor difference. One can see from the plot that the yield of dark matter rapidly grows at $T \sim m_{\phi}$ due to the resonant production of $\phi$, and then the evolution is almost frozen afterwards.

\footnotetext{
${ }^{2}$ In the current version of MicrOMEGAs, full thermal mass effects are not implemented. However it is possible to include the thermal mass only for the Higgs boson by hand without difficulty.
} 
The purple and red lines in the left panel of figure 2 show the contours in the $\left(m_{\chi}\right.$, $\left.\lambda_{\Phi}\right)$ plane reproducing the dark matter relic abundance observed by the PLANCK Collaboration [16]. The reheating temperature is assumed to be much higher than the mediator mass scale $\left(T_{R} \gg m_{\phi}\right)$. As can be seen in the plots, for a larger $m_{\phi}$ the lines simply shift to the right (purple to red), namely the direction of heavier dark matter mass which compensates a smaller yield $Y_{D}^{\mathrm{IR}}$. The behavior of the lines change around $\lambda_{\Phi} \sim \lambda_{H \Phi}$ as explained in eqs. (3.17) and (3.18). While one can take a heavier dark matter mass $m_{\chi}$ if a larger mediator mass $m_{\phi}$ is chosen, there is an upper bound on $m_{\chi}$ if the criterion for the VEV $v_{\phi}<M_{P}$ is taken into account. As we will discuss in the next section, a feeble value of the scalar self-coupling, typically $\lambda_{\Phi} \gtrsim \mathcal{O}\left(10^{-12}\right)$ is suitable for $\phi$ being the inflaton.

\subsection{UV freeze-in $\left(T_{R} \ll m_{\phi}\right)$}

When $m_{\phi}$ is much larger than the reheating temperature, the dark matter relic abundance is determined by the portal coupling $\lambda_{H \Phi}$ and the reheating temperature $T_{R}$. This is so called the UV freeze-in discussed in refs. [15, 20]. For $T<T_{R} \ll m_{\phi}$, only the $H^{\dagger} H \rightarrow \chi \chi$ process is effective for the dark matter production (see the left panel of figure 1). In the $H^{\dagger} H \rightarrow \chi \chi$ reaction rate (3.10), we can safely assume $m_{\phi} \gg \sqrt{s} \gg m_{h}, m_{\chi}$ since the modified Bessel function regarded as the window function with a cut-off $T$ and the large $s$ contribution is dominant due to the $s^{5 / 2}$ behavior of the integrand. Thus the reaction rate is approximated by

$$
\left\langle\sigma_{H^{\dagger} H \rightarrow \chi \chi} \bar{v}\right\rangle\left(n_{H}^{\mathrm{eq}}\right)^{2} \approx \frac{\lambda_{H \Phi}^{2} T}{512 \pi^{5}} \int_{0}^{\infty} d s \frac{s^{5 / 2}}{m_{\phi}^{4}} K_{1}(\sqrt{s} / T)=\frac{3 \lambda_{H \Phi}^{2} T^{8}}{2 \pi^{5} m_{\phi}^{4}} .
$$

By integrating the Boltzmann equations for the dark sector using this approximation, the pNGB dark matter yield is evaluated as

$$
Y_{D}^{\mathrm{UV}} \approx \frac{135 \sqrt{10} \lambda_{H \Phi}^{2} M_{P} T_{R}^{3}}{4 \pi^{8} g_{*}^{S} g_{*}^{1 / 2} m_{\phi}^{4}}
$$

Combining with the observed value $Y_{D}^{\text {obs. }}=4.4 \times 10^{-7}\left(m_{\chi} / \mathrm{MeV}\right)^{-1}$ with $g_{*} \sim g_{*}^{S} \sim 100$, we obtain the following relation ${ }^{3}$

$$
\left(\frac{\lambda_{H \Phi}}{10^{-6}}\right)^{2} \approx\left(\frac{1 \mathrm{MeV}}{m_{\chi}}\right)\left(\frac{m_{\phi}}{10^{5} \mathrm{GeV}}\right)^{4}\left(\frac{10^{4} \mathrm{GeV}}{T_{R}}\right)^{3} .
$$

The right panel of figure 2 shows the parameter space where the dark matter relic can be realized by the UV freeze-in, which is denoted by the purple region. The purple solid, dashed and dot-dashed lines denote $m_{\phi}=100 T_{R}, 10 T_{R}$ and $T_{R}$ (the UV freeze-in (3.22) is valid for $\left.m_{\phi} \gg T_{R}\right)$. The red lines show the contours in the $\left(m_{\chi}, \lambda_{H \Phi}\right)$ plane reproducing the dark matter relic for various given reheating temperatures. Note that the green colored region $m_{\phi} \geq 10^{-3} M_{P}$ is excluded by the conditions $v_{\phi} \leq M_{P}$ and $\lambda_{\Phi} \leq 10^{-6}$, the latter of which is required for the dark sector not being thermalized.

\footnotetext{
${ }^{3}$ For a heavier pNGB dark matter case, the broader parameter space in the $\left(m_{\phi}, \lambda_{H \Phi}\right)$ plane has been discussed in ref. [21].
} 


\section{Inflation}

In the previous section, we have seen the pNGB dark matter relic abundance realized via feeble scalar couplings, where the radial component $\phi$ of the symmetry breaking scalar plays a role of the mediator of pNGB production. In this section, we investigate the possibility that $\phi$ also plays another important role, namely, the inflaton. ${ }^{4}$

\subsection{Inflationary dynamics and constraints}

The inflation gives a plausible solution for the flatness and horizon problems in the universe. A successful inflation scenario can occur in our model if a non-minimal coupling between the complex scalar $\Phi$ and gravity is introduced. Then the Lagrangian relevant for the inflation dynamics is given by

$$
\frac{\mathcal{L}}{\sqrt{-g}}=-\frac{M_{P}^{2}}{2} \mathcal{R}-\xi|\Phi|^{2} \mathcal{R}+g^{\mu \nu}\left(\partial_{\mu} \Phi\right)^{*}\left(\partial_{\nu} \Phi\right)-\mathcal{V}(\Phi)
$$

where $\mathcal{R}$ is the Ricci scalar and $\xi$ is the so-called non-minimal coupling constant. During the inflation era, the scalar potential is assumed to be dominated by the field value of $\Phi$, thus $\mathcal{V}(\Phi) \approx \lambda_{\Phi}|\Phi|^{4} / 2 \approx \lambda_{\Phi} \phi^{4} / 8$, and the $|\Phi|^{2}$ part can be written as $|\Phi|^{2} \approx \phi^{2} / 2$ with the non-linear representation $\Phi=\left(v_{\phi}+\phi\right) e^{i \chi / v_{\phi}} / \sqrt{2}$.

The non-minimal coupling is removed by the conformal transformation,

$$
g_{\mu \nu} \rightarrow g_{\mu \nu}=\Omega^{-2} \hat{g}_{\mu \nu} \quad \text { with } \quad \Omega=\sqrt{1+\frac{\xi \phi^{2}}{M_{P}^{2}}}
$$

where $\hat{g}_{\mu \nu}$ corresponds to the metric in the Einstein frame. As a result of this transformation, the Lagrangian becomes

$$
\frac{\hat{\mathcal{L}}}{\sqrt{-\hat{g}}}=-\frac{M_{P}^{2}}{2} \hat{\mathcal{R}}+\frac{\hat{g}^{\mu \nu}}{2}\left(\partial_{\mu} \varphi\right)\left(\partial_{\nu} \varphi\right)+\frac{\hat{g}^{\mu \nu}}{2}\left(\partial_{\mu} \hat{\chi}\right)\left(\partial_{\nu} \hat{\chi}\right)-\hat{\mathcal{V}}(\varphi)
$$

where $\varphi$ and $\hat{\chi}$ are the canonically normalized fields satisfying the differential equations

$$
\frac{d \varphi}{d \phi}=\sqrt{\frac{M_{P}^{2} \Omega^{2}+6 \xi^{2} \phi^{2}}{M_{P}^{2} \Omega^{4}}}, \quad \frac{d \hat{\chi}}{d \chi}=\Omega^{-1}
$$

If $\xi \gg 1$, which is similar to the case of the Higgs inflation [24], the differential equation is simplified and the explicit expressions of $\varphi$ and $\hat{\mathcal{V}}(\varphi)$ are obtained. However in the present pNGB model with feeble couplings, we will show later that a smaller $\xi$ is favored for successful inflation. The shape of the scalar potential is numerically evaluated and shown in figure 3 where the non-minimal coupling is fixed to be $\xi=10^{-2}, 10^{-1}, 1$. The flat part of the potential gets longer for a smaller value of $\xi$.

\footnotetext{
${ }^{4}$ Some different types of models are studied in the literature, e.g. [22, 23] about possible common origins of the inflaton and dark matter.
} 


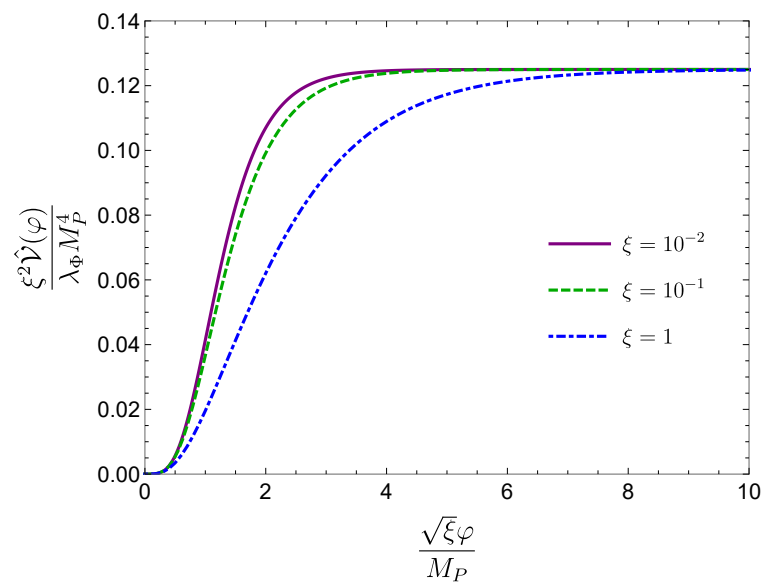

Figure 3. Inflation potential with the non-minimal coupling $\xi=10^{-2} 10^{-1}$, and 1 .

While one cannot write down the explicit form of the scalar potential $\hat{\mathcal{V}}(\varphi)$, the slowroll parameters are expressed in terms of $\phi$,

$$
\begin{aligned}
\epsilon & \equiv \frac{M_{P}^{2}}{2}\left(\frac{\hat{\mathcal{V}}_{\varphi}}{\hat{\mathcal{V}}}\right)^{2}=\frac{8 M_{P}^{4}}{\phi^{2}\left[M_{P}^{2}+\xi(1+6 \xi) \phi^{2}\right]}, \\
\eta & \equiv M_{P}^{2} \frac{\hat{\mathcal{V}}_{\varphi \varphi}}{\hat{\mathcal{V}}}=\frac{4 M_{P}^{2}\left[3 M_{P}^{4}+M_{P}^{2} \xi(1+12 \xi) \phi^{2}-2 \xi^{2}(1+6 \xi) \phi^{4}\right]}{\phi^{2}\left[M_{P}^{2}+\xi(1+6 \xi) \phi^{2}\right]^{2}}
\end{aligned}
$$

where $\hat{\mathcal{V}}_{\varphi} \equiv \partial \hat{\mathcal{V}} / \partial \varphi$ and $\hat{\mathcal{V}}_{\varphi \varphi} \equiv \partial^{2} \hat{\mathcal{V}} / \partial \varphi^{2}$. Using the slow-roll approximation, the spectral index $n_{s}$ and the tensor-to-scalar ratio $r$ are given by

$$
n_{s}=1-6 \epsilon+2 \eta, \quad r=16 \epsilon .
$$

The e-folding number $N_{*}$ between the time of horizon exit $\left(t_{*}\right)$ and the end of inflation $\left(t_{\text {end }}\right)$ is given by

$$
N_{*}=\int_{t_{*}}^{t_{\text {end }}} H d t \approx \frac{-1}{M_{P}^{2}} \int_{\phi_{*}}^{\phi_{\text {end }}} \frac{d \varphi}{d \phi} \frac{\hat{\mathcal{V}}}{\hat{\mathcal{V}}_{\varphi}} d \phi=\frac{(1+6 \xi)\left(\phi_{*}^{2}-\phi_{\mathrm{end}}^{2}\right)}{8 M_{P}^{2}}-\frac{3}{4} \log \left(\frac{M_{P}^{2}+\xi \phi_{*}^{2}}{M_{P}^{2}+\xi \phi_{\mathrm{end}}^{2}}\right),
$$

where $\phi_{*}$ and $\phi_{\text {end }}$ are the field values at $t_{*}$ and $t_{\text {end }}$, respectively. Defining $t_{\text {end }}$ as the time giving the slow-roll parameter $\epsilon=1$ in eq. (4.5), $\phi_{\text {end }}$ satisfies

$$
\phi_{\text {end }}^{2}=\frac{M_{P}^{2}}{2} \frac{\sqrt{1+32 \xi+192 \xi^{2}}-1}{\xi(1+6 \xi)} .
$$

On the other hand, $\phi_{*}$ is numerically evaluated, giving the amplitude of the scalar power spectrum at the horizon exit observed by the Planck Collaboration [25]: $A_{s}=$ $\hat{\mathcal{V}}\left(\varphi\left(\phi_{*}\right)\right) /\left(24 \pi^{2} M_{P}^{4} \epsilon\left(\phi_{*}\right)\right)=2.10 \times 10^{-9}$. From these relations, the non-minimal coupling $\xi$ and the scalar self-coupling $\lambda_{\Phi}$ for successful inflation can be read. The contour of the efolding number $N_{*}$ on the $\left(\xi, \lambda_{\Phi}\right)$ plane is shown in the left panel of figure 4 where $N_{*}=50$ 

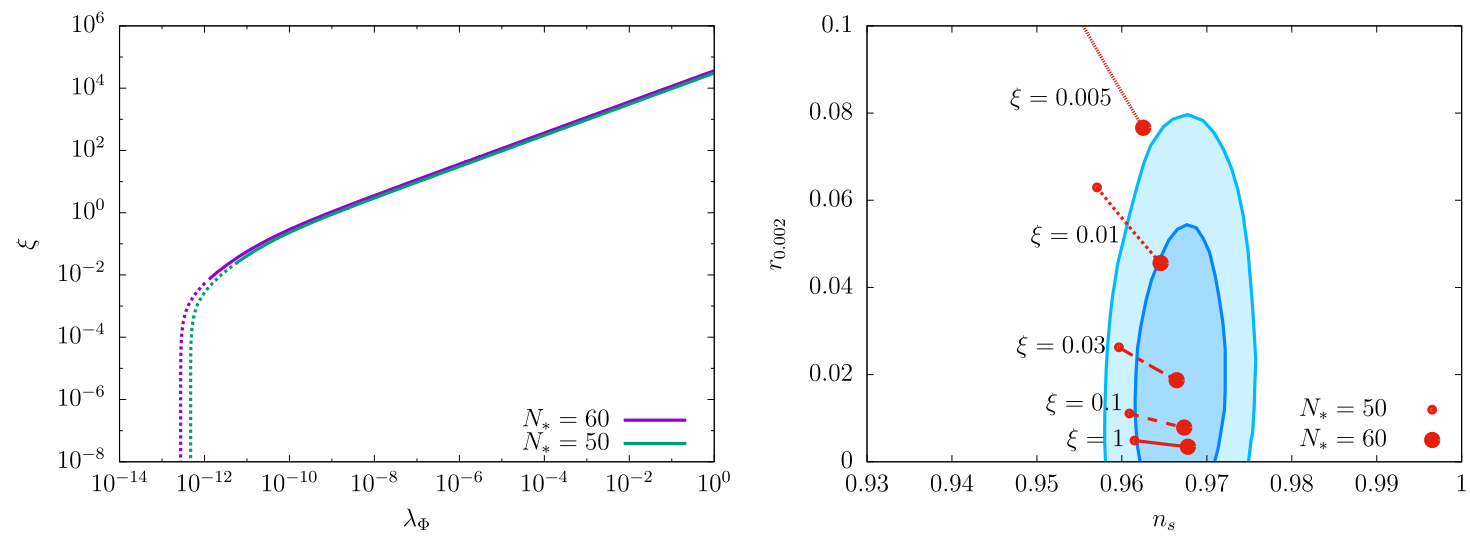

Figure 4. Left: contours in the $\left(\xi, \lambda_{\Phi}\right)$ plane for successful inflation where $N_{*}$ is fixed as $N_{*}=50$ and 60. Right: predictions for the spectral index $\left(n_{s}\right)$ and the tensor-to-scalar ratio $(r)$ where the non-minimal coupling $\xi$ is taken as $\xi=1,0.1,0.03,0.01$ and 0.005 . The blue and light blue regions are allowed by the PLANCK observation at $1 \sigma$ and $2 \sigma$ confidence level, respectively.

and 60 . When the non-minimal coupling $\xi$ is small enough $\left(\xi \lesssim 10^{-3}\right)$, the dependence on $\lambda_{\Phi}$ disappears. This region has the same behavior as the $\phi^{4}$ chaotic inflation.

The predictions for the spectral index $n_{s}$ and the tensor-to-scalar ratio $r$ at the pivot scale $k_{*}=0.002 \mathrm{Mpc}^{-1}$ are shown in the right panel of figure 4 where $N_{*}$ is taken to be between 50 and 60 . The blue and light blue regions represent the $1 \sigma$ and $2 \sigma$ confidence levels observed by the Planck Collaboration [25]. It can be found that the lower bounds for the non-minimal coupling are required

$$
\xi \gtrsim 0.02 \quad\left(N_{*}=50\right), \quad \xi \gtrsim 0.0055 \quad\left(N_{*}=60\right),
$$

for the scenario consistent with the observation.

In the left panel of figure 4, the solid (dotted) part of each contour represents the parameter region consistent (inconsistent) with the PLANCK observation at $2 \sigma$ confidence level, when combined with the lower bounds of $\xi$ obtained in the right panel. We find that the self-coupling $\lambda_{\Phi}$ should be in the range $\lambda_{\Phi} \gtrsim 10^{-12}$ in the pNGB dark matter model with large symmetry breaking, if the inflation is induced by the coupling $\xi$.

\section{2 pNGB production from inflaton}

There is another important physical implication of the possibility that the radial scalar component $\phi$ plays the role of inflaton in the pNGB dark matter model. That is the direct production process of dark matter $\chi$ from the inflaton decay, which is inevitable because of the interaction between $\phi$ and $\chi$. In this section, we investigate the parameter space consistent with the inflationary scenario discussed above and the dark matter relic abundance taking into account both the freeze-in and inflaton-induced dark matter.

As discussed in refs. [26, 27], the number density of the inflaton induced $\chi$ particle is estimated as

$$
n_{\chi}^{\inf } \approx \frac{\rho_{\mathrm{rh}}}{m_{\phi}} \frac{\Gamma_{\phi \rightarrow \chi \chi}}{\sqrt{3} H\left(T_{R}\right)}
$$



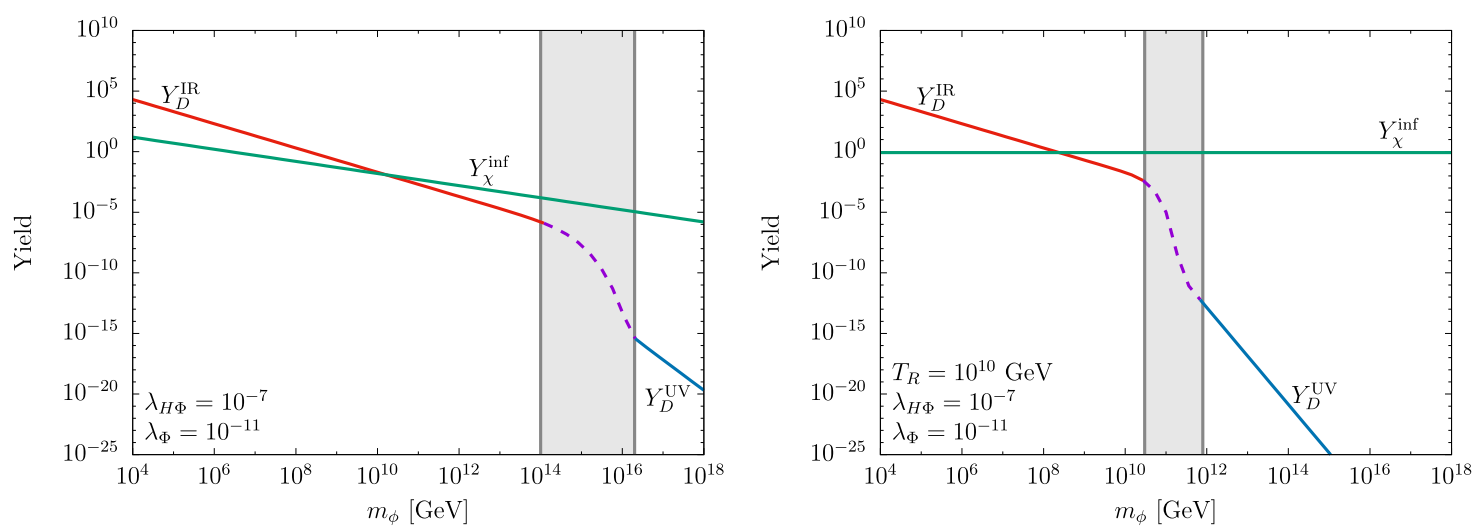

Figure 5. Comparisons of the pNGB dark matter yields, $Y_{D}^{\mathrm{IR}}$ (red), $Y_{D}^{\mathrm{UV}}$ (green) and $Y_{\chi}^{\mathrm{inf}}$ (blue) for $\lambda_{H \Phi}=10^{-7}$ and $\lambda_{\Phi}=10^{-11}$. The reheating temperature is given by eq. (4.12) in the left panel and a free parameter in the right panel, chosen as $T_{R}=10^{10} \mathrm{GeV}$. The IR and UV freeze-in are smoothly connected in the gray region, typically $T_{R} \lesssim m_{\phi} \lesssim 100 T_{R}$.

where $\rho_{\text {rh }}$ and $H\left(T_{R}\right)$ are the energy density of radiation and the Hubble rate at the reheating temperature $T_{R}$. We here define the reheating temperature $T_{R}$ at which the Hubble rate is equal to the decay width of the inflaton to the SM sector $\left(H\left(T_{R}\right) \approx \Gamma_{\phi \rightarrow H^{\dagger} H}\right)$, and then

$$
T_{R}^{2} \approx \frac{3 \sqrt{10}}{8 \pi^{2} g_{*}^{1 / 2}} \frac{\lambda_{H \Phi}^{2}}{\lambda_{\Phi}} M_{P} m_{\phi} \sqrt{1-\frac{4 m_{h}^{2}}{m_{\phi}^{2}}} .
$$

Note that the effective degrees of freedom $g_{*}$ in the right-hand side also depends on the temperature in general. Using the explicit form of the decay widths and the reheating temperature (4.12), we obtain the pNGB dark matter yield directly produced from the inflaton as

$$
Y_{\chi}^{\inf } \approx\left(\frac{9 \sqrt{10}}{2048 \pi^{2}}\right)^{1 / 2} \frac{g_{*}^{3 / 4} \lambda_{\Phi}^{3 / 2} M_{P}^{1 / 2}}{g_{*}^{S} \lambda_{H \Phi} m_{\phi}^{1 / 2}}\left(1-\frac{4 m_{\chi}^{2}}{m_{\phi}^{2}}\right)^{\frac{1}{2}}\left(1-\frac{4 m_{h}^{2}}{m_{\phi}^{2}}\right)^{-\frac{1}{4}}
$$

As previously, we impose the portal coupling satisfies $\lambda_{H \Phi} \lesssim 10^{-6}$ such that dark matter does not get into the SM thermal bath.

We here comment on the comparison between the three contributions of pNGB dark matter yields, $Y_{D}^{\mathrm{IR}}, Y_{D}^{\mathrm{UV}}$ and $Y_{\chi}^{\mathrm{inf}}$. Typical behaviors are shown in figure 5 as the functions of the mediator mass $m_{\phi}$, which behaviors are evaluated by MicrOMEGAs for $\lambda_{H \Phi}=10^{-7}$ and $10^{-11}$. The reheating temperature is given by eq. (4.12) in the left panel, while it is treated as a free parameter in the right panel (see the detail in the next subsection). The IR and UV freeze-in productions are effective in the smaller and larger $m_{\phi}$ regions, respectively. These freeze-in yields are smoothly connected in the gray region where $T_{R} \lesssim m_{\phi} \lesssim 100 T_{R}$, which is denoted by the purple dashed line. Since $Y_{D}^{\mathrm{IR}} \propto m_{\phi}^{-1}$ and $Y_{\chi}^{\mathrm{inf}} \propto m_{\phi}^{-1 / 2}$, the IR freeze-in is dominant for a lighter mediator. Further, $Y_{D}^{\mathrm{IR}}$ becomes equal to $Y_{\chi}^{\mathrm{inf}}$ at $m_{\phi}=$ $m_{\text {eq }}$. It is easy to find from eqs. (3.15), (4.12) and (4.13) that $m_{\text {eq }} \lesssim 10^{-2} T_{R}$, and then 

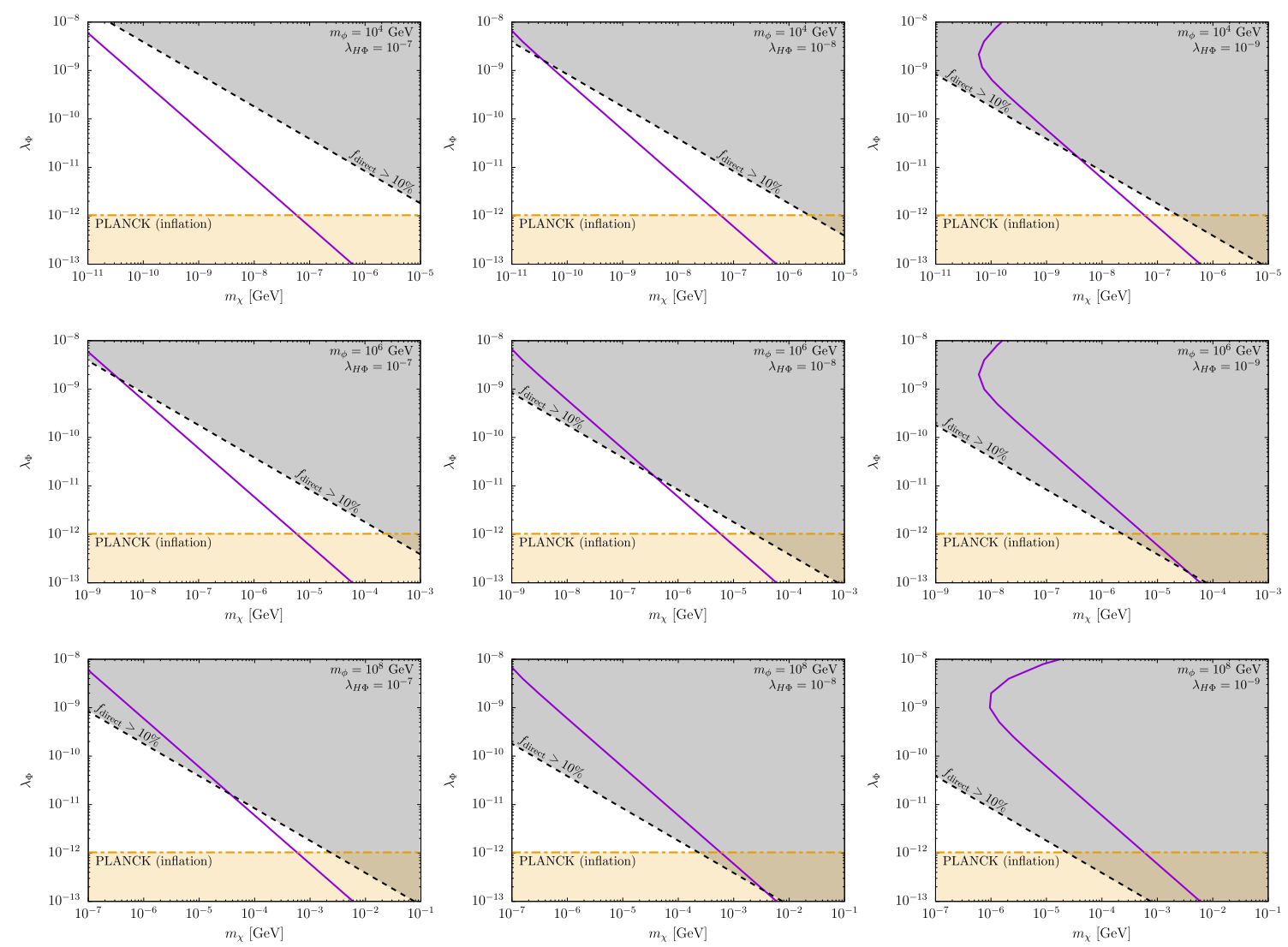

Figure 6. Parameter space in the $\left(m_{\chi}, \lambda_{\Phi}\right)$ plane where the reheating of the universe is assumed to occur via the perturbative inflaton decay into the SM sector. The purple lines reproduce the correct relic abundance of dark matter only by the freeze-in production. The gray regions represent the parameter space that the dark matter abundance created by the inflaton decay is larger than $10 \%$ of the observed value. The lower orange region cannot be consistent with the inflation observables at $2 \sigma$ confidence level.

the inflaton-induced yield necessarily becomes dominant in the left side of the gray band. On the other hand, the UV freeze-in abundance takes the value $Y_{D}^{\mathrm{UV}} / Y_{\chi}^{\mathrm{inf}} \simeq 10^{-3} \lambda_{H \Phi}^{2} / \lambda_{\Phi}$ around $m_{\phi}=T_{R}$. This ratio is much smaller than 1 due to the constraints $\lambda_{H \Phi} \lesssim 10^{-6}$ and $\lambda_{\Phi} \gtrsim 10^{-12}$ as we explained from the cosmological arguments. Since $Y_{D}^{\mathrm{UV}} \propto m_{\phi}^{-5 / 2}$, the UV freeze-in contribution is always subdominant compared to the inflaton decay.

\subsection{Dark matter abundance}

Figure 6 shows the parameter space realizing the correct dark matter relic abundance in the $\left(m_{\chi}, \lambda_{\Phi}\right)$ plane taking into account the pNGB production from the freeze-in and inflaton decay. The portal coupling is chosen as $\lambda_{H \Phi}=10^{-7}, 10^{-8}$ and $10^{-9}$ and the mediator mass is $m_{\phi}=10^{4} \mathrm{GeV}, 10^{6} \mathrm{GeV}$ and $10^{8} \mathrm{GeV}$. The purple line represents the parameters reproducing the dark matter abundance only by the freeze-in contribution. The gray region represents the parameter space where the inflaton induced dark matter abundance is larger than $10 \%$ of the observed value. In order to be consistent with the inflation observables 

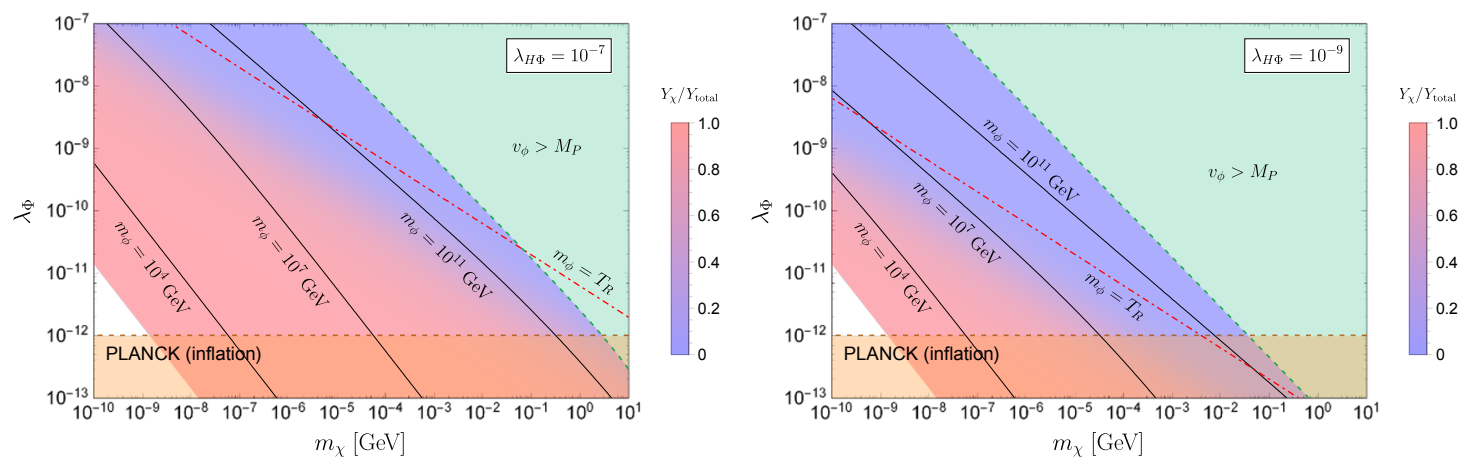

Figure 7. Parameter space in the $\left(m_{\chi}, \lambda_{\Phi}\right)$ plane where the correct relic abundance of dark matter is realized by the non-thermal production (the freeze-in and the inflaton decay). The portal coupling is chosen as $\lambda_{H \Phi}=10^{-7}$ (left) and $\lambda_{H \Phi}=10^{-9}$ (right). The freeze-in production is dominant in the red region and the inflaton decay dominant in the blue region. The lower orange region is not consistent with the inflation observables as in figure 6. In the green region, the scalar VEV becomes trans-Planckian.

at $2 \sigma$ confidence level as discussed in the previous subsection, the scalar self-coupling has to satisfy $\lambda_{\Phi} \gtrsim 10^{-12}$ and the lower orange region is excluded by this condition.

The behavior of the purple lines in the most of panels can be understood by the IR freeze-in as discussed in section 3.3, except the right-bottom one. The region $\lambda_{\Phi} \gtrsim$ $10^{-9}$ of that plot corresponds to the intermediate state between the IR and UV freeze-in, namely $T_{R} \sim m_{\phi}$, while the contribution to the relic from the inflaton decay is eventually dominant in this region. In case that the reheating temperature determined by the inflaton decay is much lower than the mediator mass $m_{\phi}$, since the reheating temperature scales as $T_{R} \propto \lambda_{H \Phi} \lambda_{\Phi}^{-1 / 2} m_{\phi}^{1 / 2}$ which can be seen in eq. (4.12), it finds that the self-coupling goes as $\lambda_{\Phi} \propto m_{\chi}^{2 / 3}$ to reproduce the correct relic abundance from eq. (3.23) for the UV freeze-in. This behavior has been numerically checked in our computation.

Figure 7 shows the parameter space in the $\left(m_{\chi}, \lambda_{\Phi}\right)$ plane realizing the correct dark matter relic abundance via both contributions from the freeze-in and inflaton induced productions. The red and blue regions mean the abundance is dominated by the freeze-in and the inflaton, respectively. The red dot-dashed line denotes $m_{\phi}=T_{R}$. In the green region, the singlet scalar VEV $\left(v_{\phi}\right)$ becomes trans-Planckian, where the low-energy field description is not valid. ${ }^{5}$ When the dark sector scalars $\phi$ and/or $\chi$ are heavy, the inflaton decay tends to be the dominant process for the dark matter creation. A physical implication of figure 7 is that in the pNGB dark matter model with large symmetry breaking, the dark matter should be lighter than $\mathrm{MeV}-\mathrm{GeV}$ if the freeze-in production is assumed to be dominant. A heavier dark matter is also possible if taking the inflaton induced contribution into account.

\footnotetext{
${ }^{5}$ When one considers graviton loop effect, its form may be $\frac{m^{2}}{16 \pi^{2} M_{P}^{2}}$ where $m$ is a typical scale in lowenergy theory. Therefore the low-energy perturbative description is violated above $m_{P} \simeq 4 \pi M_{P}$. If one imposes the condition $v_{\phi}<m_{P}$ in figure 7 , the green excluded region is relaxed by $4 \pi$.
} 

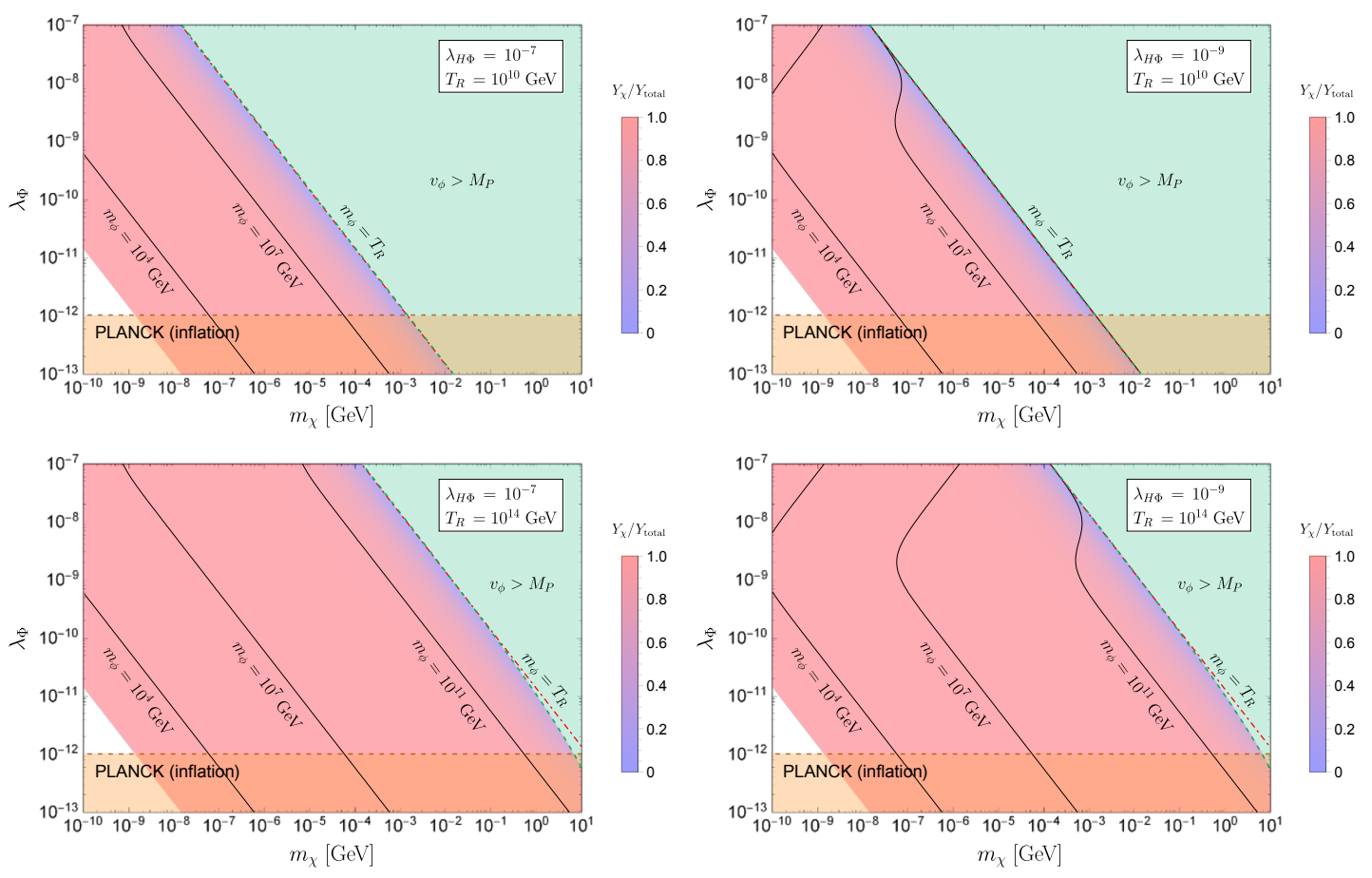

Figure 8. Same plots as figure 7 while the reheating temperature is taken to be a free parameter. The portal coupling is chosen as $\lambda_{H \Phi}=10^{-7}$ (left) and $10^{-9}$ (right), and the reheating temperature is $T_{R}=10^{10} \mathrm{GeV}$ (above) and $10^{14} \mathrm{GeV}$ (below).

In the above analysis, we have assumed that the reheating of the universe simply occurs via the inflaton perturbative decay to the Higgs field. However if there exists some other decay modes of the inflaton, the reheating temperature generally takes a different value. In this situation, the inflaton induced dark matter is estimated by

$$
Y_{\chi}^{\mathrm{inf}} \approx \frac{3 \sqrt{30}}{128 \pi^{2}} \frac{g_{*}^{1 / 2} \lambda_{\Phi} M_{P}}{g_{*}^{S} T_{R}} \sqrt{1-\frac{4 m_{\chi}^{2}}{m_{\phi}^{2}}},
$$

which is independent of the Higgs portal coupling $\lambda_{H \Phi}$ and almost independent of $m_{\phi}$ for a relatively heavy $\phi$. The pNGB abundance from the UV freeze-in is also implicitly changed since the parameter dependence of $T_{R}$ is modified from eq. (4.12) to a free parameter, though the formula of $Y_{D}^{\mathrm{UV}}$ is still valid. In the same fashion in figure 7, we draw the parameter space realizing the correct dark matter abundance via both of freeze-in and inflaton decay (figure 8). We find in almost all allowed regions the freeze-in production is dominant. ${ }^{6}$ This comes from the fact that the inflaton induced abundance eq. (4.14) is almost insensitive to $m_{\phi}$. Compared with figure 7, a heavier dark matter is possible up to $\mathcal{O}(10-100) \mathrm{GeV}$ with a higher reheating temperature.

\footnotetext{
${ }^{6}$ The light dark matter is constrained from the dark matter free streaming length being inconsistent with measurements of the Lyman-alpha forest [28, 29]. This typically excludes the dark matter mass region lighter than $\mathrm{keV}$, which gives the lower mass bound.
} 


\section{Conclusion}

The pNGB dark matter model has been originally motivated from the fact that the strong constraint of direct detection can naturally be evaded even when it is a WIMP with sufficiently large couplings with the SM particles. On the other hand, the pNGB dark matter can also be regarded as a natural FIMP candidate if the VEV of the symmetry-breaking scalar is large enough. That is because all couplings of the pNGB are suppressed by the large VEV due to its NG property.

We have studied the model parameters for which the dark matter relic is reproduced by the feeble couplings of pNGB, taking into account the effect of thermal mass of the Higgs field. The dark matter relic abundance is mainly determined by the mediator mass and a smaller coupling of $\lambda_{\Phi}$ and $\lambda_{H \Phi}$ when the reheating temperature of the universe is larger than the mediator mass. On the contrary, the abundance depends on the reheating temperature if it is not large as the mediator mass. These feature are similar to typical FIMPs.

We have also investigated the possibility that the radial component of the symmetrybreaking scalar $\Phi$ plays a role of the inflaton. Introducing the non-minimal coupling of $\Phi$ to gravity, the flat potential is understood by the rescaling, and the parameter space consistent with the observations has been explored. We have found that the scalar self coupling is tiny $\lambda_{\Phi} \gtrsim 10^{-12}$ and the non-minimal coupling should be $\xi \gtrsim 10^{-2}$, which are rather different from the Higgs inflation scenario. Furthermore, it is important the inflaton decay into the pNGB is unavoidable. We have examined the allowed parameter regions taking into account both of the freeze-in and the inflaton decay. Combining these requirements for the dark matter relic and the successful inflation, it is found that the pNGB FIMP dark matter should be lighter than a few $\mathrm{GeV}$ when the freeze-in contribution is assumed to be dominant. A heavier pNGB dark matter with $\mathcal{O}\left(10^{1-2}\right) \mathrm{GeV}$ mass is possible if the inflaton-induced contribution comes to be effective and/or the reheating process depends on some other dynamics.

In the present model, since the pNGB dark matter is stable due to the $\mathbb{Z}_{2}$ symmetry: $\chi \mapsto-\chi$ coming from the CP invariance of the scalar potential, one may feel there is no detectable signals from the pNGB dark matter with feeble couplings. However if the remnant symmetry is not exact as easily expected in a UV completion of the model [4], the pNGB can decay into lighter SM particles. Even if its couplings are highly suppressed by the large VEV, some signals may be detectable in cosmic-ray observations. That is left for future study.

\section{Acknowledgments}

The authors thank Geneviève Bélanger, Tetsutaro Higaki, Takahiro Ohata and Alexander Pukhov for the useful discussions and comments. The numerical computation in this work was carried out at the Yukawa Institute Computer Facility. This work is supported by JSPS Grant-in-Aid for Scientific Research KAKENHI Grant No. JP20J11901 (YA), JP20K22349 (TT), JP18H01214 and JP20K03949 (KY). 


\section{A Thermal mass contribution}

Referring to [30], we summarize the derivation of thermal mass and its formula in the high temperature era.

We consider a field variable decomposed to the background configuration $\sigma$ and its fluctuation $\rho$, and integrate out the latter. Then the one-loop effective potential for the background $\sigma$ is given by

$$
\mathcal{V}_{\mathrm{eff}}^{T}(\sigma)=\mathcal{V}_{0}(\sigma)+\mathcal{V}_{1}(\sigma)+\mathcal{V}_{1}^{T}(\sigma ; T)
$$

where $\mathcal{V}_{0}(\sigma)$ is the classical potential for $\sigma$, and $\mathcal{V}_{1}(\sigma), \mathcal{V}_{1}^{T}(\sigma)$ are the one-loop contributions. According to refs. [31, 32], the effective potential is evaluated on $\mathbb{R}^{3} \times S^{1}$ with the radius $1 / T$ in order to take the thermal effect into account.

The effective potential for a real scalar is expressed as

$$
\mathcal{V}_{1 B}(\sigma)=\int \frac{d^{3} k}{(2 \pi)^{3}} \frac{E_{k}}{2}, \quad \mathcal{V}_{1 B}^{T}(\sigma ; T)=\frac{T^{4}}{2 \pi^{2}} J_{B}(M / T),
$$

where $E_{\boldsymbol{k}}^{2}=\boldsymbol{k}^{2}+M^{2}$, and $M$ denotes the $\rho$ mass in the $\sigma$ background. The mass $M$ is typically given by $M^{2}=M(\sigma)^{2}=m_{b}^{2}+\frac{\lambda}{2} \sigma^{2}$ for the $\rho$ mass $m_{b}$ and the scalar self quartic coupling $\lambda$. The function $J_{B}(y)$ is given by

$$
J_{B}(y)=\int_{0}^{\infty} d x x^{2} \log \left(1-e^{-\left(x^{2}+y^{2}\right)^{1 / 2}}\right) .
$$

In the high-temperature region corresponding to $y \ll 1$, the function $J_{B}(y)$ is approximately written as

$$
J_{B}(y) \approx-\frac{\pi^{4}}{45}+\frac{\pi^{2}}{12} y^{2}-\frac{\pi}{6} y^{3}-\frac{y^{4}}{32} \log \left(\frac{y^{2}}{a_{B}}\right)
$$

with $a_{B}=\pi^{2} e^{3 / 2-2 \gamma_{E}}$ and $\gamma_{E}$ is the Euler constant. The $y^{2}$ term contributes to the thermal mass.

For a Dirac fermion, the one-loop effective potential is given by

$$
\mathcal{V}_{1 F}(\sigma)=-4 \int \frac{d^{3} k}{(2 \pi)^{3}} \frac{E_{\boldsymbol{k}}}{2}, \quad \mathcal{V}_{1 F}^{T}(\sigma ; T)=-\frac{2 T^{4}}{\pi^{2}} J_{F}(M / T),
$$

with

$$
J_{F}(y)=-\int_{0}^{\infty} d x x^{2} \log \left(1+e^{-\left(x^{2}+y^{2}\right)^{1 / 2}}\right)
$$

The mass in the $\sigma$ background is typically given by using a Yukawa coupling $g$ as $M=$ $M(\sigma)=m_{f}+g \frac{\sigma}{\sqrt{2}}$, for the fermion mass $m_{f}$. As in the bosonic case, the function $J_{F}(y)$ is approximately written as

$$
J_{F}(y) \approx \frac{7 \pi^{4}}{360}-\frac{\pi^{2}}{24} y^{2}-\frac{y^{4}}{32} \log \left(\frac{y^{4}}{a_{F}}\right)
$$

with $a_{F}=16 \pi^{2} e^{3 / 2-2 \gamma_{E}}$ in the high-temperature region corresponding to $y \ll 1$. 
The thermal mass is defined from the one-loop effective potential $\mathcal{V}_{1}^{T}(\sigma ; T)$ as

$$
\left.\Delta \equiv \frac{\partial^{2} \mathcal{V}_{1}^{T}}{\partial \sigma^{2}}\right|_{\sigma=0}
$$

Using the above formulae, the thermal mass contributions of particle $i$ are given by

$$
\begin{cases}\Delta^{B}=g_{i} \frac{T^{4}}{2 \pi^{2}} \frac{\pi^{2}}{12} \frac{M^{2}(\sigma)^{\prime \prime}}{T^{2}}=\frac{g_{i} T^{2} M^{2}(\sigma)^{\prime \prime}}{24} & \text { for bosons } \\ \Delta^{F}=-g_{i} \frac{T^{4}}{2 \pi^{2}}\left(\frac{-\pi^{2}}{24}\right) \frac{M^{2}(\sigma)^{\prime \prime}}{T^{2}}=\frac{g_{i} T^{2} M^{2}(\sigma)^{\prime \prime}}{48} & \text { for fermions }\end{cases}
$$

where $g_{i}$ is the degrees of freedom of the particle $i$ and the prime means the derivative with respect to $\sigma$.

For the SM Higgs boson, there are three sources of thermal mass; electroweak gauge bosons, quarks and leptons, and Higgs scalar. Applying the above result to the SM, the thermal mass contributions are found

$$
\begin{aligned}
\Delta_{h}^{\text {gauge }} & =\frac{g_{Y}^{2}}{16} T^{2}+\frac{3 g_{2}^{2}}{16} T^{2}, \\
\Delta_{h}^{\text {fermion }} & =\frac{T^{2}}{12}\left[y_{e}^{2}+y_{\mu}^{2}+y_{\tau}^{2}+3\left(y_{u}^{2}+y_{d}^{2}+y_{c}^{2}+y_{s}^{2}+y_{t}^{2}+y_{b}^{2}\right)\right] \approx \frac{y_{t}^{2}}{4} T^{2}, \\
\Delta_{h}^{\text {scalar }} & =\frac{\lambda_{H}}{4} T^{2} .
\end{aligned}
$$

Open Access. This article is distributed under the terms of the Creative Commons Attribution License (CC-BY 4.0), which permits any use, distribution and reproduction in any medium, provided the original author(s) and source are credited.

\section{References}

[1] XENON collaboration, Dark matter search results from a one ton-year exposure of XENON1T, Phys. Rev. Lett. 121 (2018) 111302 [arXiv:1805.12562] [InSPIRE].

[2] XENON collaboration, Projected WIMP sensitivity of the XENONnT dark matter experiment, JCAP 11 (2020) 031 [arXiv: 2007.08796] [INSPIRE].

[3] C. Gross, O. Lebedev and T. Toma, Cancellation mechanism for dark-matter-nucleon interaction, Phys. Rev. Lett. 119 (2017) 191801 [arXiv:1708. 02253] [INSPIRE].

[4] Y. Abe, T. Toma and K. Tsumura, Pseudo-Nambu-Goldstone dark matter from gauged $\mathrm{U}(1)_{B-L}$ symmetry, JHEP 05 (2020) 057 [arXiv: 2001.03954] [INSPIRE].

[5] N. Okada, D. Raut and Q. Shafi, Pseudo-Goldstone dark matter in gauged B $-L$ extended standard model, arXiv:2001.05910 [INSPIRE].

[6] C. Arina, A. Beniwal, C. Degrande, J. Heisig and A. Scaffidi, Global fit of pseudo-Nambu-Goldstone dark matter, JHEP 04 (2020) 015 [arXiv:1912.04008] [INSPIRE].

[7] Y. Chikashige, R.N. Mohapatra and R.D. Peccei, Spontaneously broken lepton number and cosmological constraints on the neutrino mass spectrum, Phys. Rev. Lett. 45 (1980) 1926 [INSPIRE]. 
[8] Y. Chikashige, R.N. Mohapatra and R.D. Peccei, Are there real goldstone bosons associated with broken lepton number?, Phys. Lett. B 98 (1981) 265 [InSPIRE].

[9] G.B. Gelmini and M. Roncadelli, Left-handed neutrino mass scale and spontaneously broken lepton number, Phys. Lett. B 99 (1981) 411 [INSPIRE].

[10] P.-H. Gu, E. Ma and U. Sarkar, Pseudo-Majoron as dark matter, Phys. Lett. B 690 (2010) 145 [arXiv: 1004.1919] [INSPIRE].

[11] S. Matsumoto and K. Yoshioka, Deep correlation between cosmic-ray anomaly and neutrino masses, Phys. Rev. D 82 (2010) 053009 [arXiv:1006.1688] [InSPIRE].

[12] F.S. Queiroz and K. Sinha, The poker face of the Majoron dark matter model: LUX to keV line, Phys. Lett. B 735 (2014) 69 [arXiv:1404.1400] [INSPIRE].

[13] T. Asaka, K. Ishiwata and T. Moroi, Right-handed sneutrino as cold dark matter, Phys. Rev. D 73 (2006) 051301 [hep-ph/0512118] [INSPIRE].

[14] T. Asaka, K. Ishiwata and T. Moroi, Right-handed sneutrino as cold dark matter of the universe, Phys. Rev. D 75 (2007) 065001 [hep-ph/0612211] [INSPIRE].

[15] L.J. Hall, K. Jedamzik, J. March-Russell and S.M. West, Freeze-in production of FIMP dark matter, JHEP 03 (2010) 080 [arXiv: 0911.1120] [INSPIRE].

[16] Planck collaboration, Planck 2018 results. VI. Cosmological parameters, Astron. Astrophys. 641 (2020) A6 [arXiv: 1807.06209] [InSPIRE].

[17] E.W. Kolb and M.S. Turner, The early universe, Front. Phys. 69 (1990) 1 [InSPIRE].

[18] O. Lebedev and T. Toma, Relativistic freeze-in, Phys. Lett. B 798 (2019) 134961 [arXiv: 1908.05491] [INSPIRE].

[19] G. Bélanger, F. Boudjema, A. Goudelis, A. Pukhov and B. Zaldivar, MicrOMEGAs5.0: freeze-in, Comput. Phys. Commun. 231 (2018) 173 [arXiv:1801.03509] [inSPIRE].

[20] F. Elahi, C. Kolda and J. Unwin, Ultra Violet freeze-in, JHEP 03 (2015) 048 [arXiv:1410.6157] [INSPIRE].

[21] Y. Abe, Y. Hamada, T. Ohata, K. Suzuki and K. Yoshioka, TeV-scale Majorogenesis, JHEP 07 (2020) 105 [arXiv: 2004.00599] [INSPIRE].

[22] S.M. Boucenna, S. Morisi, Q. Shafi and J.W.F. Valle, Inflation and Majoron dark matter in the seesaw mechanism, Phys. Rev. D 90 (2014) 055023 [arXiv: 1404.3198] [INSPIRE].

[23] K. Enqvist, S. Nurmi, T. Tenkanen and K. Tuominen, Standard model with a real singlet scalar and inflation, JCAP 08 (2014) 035 [arXiv:1407.0659] [INSPIRE].

[24] F.L. Bezrukov and M. Shaposhnikov, The standard model Higgs boson as the inflaton, Phys. Lett. B 659 (2008) 703 [arXiv:0710.3755] [INSPIRE].

[25] Planck collaboration, Planck 2018 results. X. Constraints on inflation, Astron. Astrophys. 641 (2020) A10 [arXiv:1807.06211] [INSPIRE].

[26] F. Takahashi, Gravitino dark matter from inflaton decay, Phys. Lett. B 660 (2008) 100 [arXiv:0705.0579] [INSPIRE].

[27] D.S. Gorbunov and A.G. Panin, Scalaron the mighty: producing dark matter and baryon asymmetry at reheating, Phys. Lett. B 700 (2011) 157 [arXiv:1009.2448] [INSPIRE]. 
[28] M. Viel, G.D. Becker, J.S. Bolton and M.G. Haehnelt, Warm dark matter as a solution to the small scale crisis: new constraints from high redshift Lyman- $\alpha$ forest data, Phys. Rev. D 88 (2013) 043502 [arXiv:1306.2314] [INSPIRE].

[29] J. Baur, N. Palanque-Delabrouille, C. Yèche, C. Magneville and M. Viel, Lyman- $\alpha$ forests cool warm dark matter, JCAP 08 (2016) 012 [arXiv: 1512.01981] [INSPIRE].

[30] A. Katz and M. Perelstein, Higgs couplings and electroweak phase transition, JHEP 07 (2014) 108 [arXiv: 1401.1827] [INSPIRE].

[31] L. Dolan and R. Jackiw, Symmetry behavior at finite temperature, Phys. Rev. D 9 (1974) 3320 [INSPIRE].

[32] S. Weinberg, Gauge and global symmetries at high temperature, Phys. Rev. D 9 (1974) 3357 [INSPIRE]. 